\title{
HEURISTIC APPROACH FOR POWER SYSTEM OPTIMIZATION BY COMPARING THE ACTIVE AND REACTIVE POWER LOSS IN DIFFERENT BUS SYSTEMS
}

\section{Bhalchandra Tiwari}

Dr. A. K. Sharma* Master of Engineering High Voltage and Power System Engineering
Jabalpur Engineering College, Jabalpur (M.P.)

Head of the Department Electrical Engineering Department Jabalpur Engineering College, Jabalpur (M.P.) *Corresponding Author

ABSTRACT Power flow is basically the flow of active and reactive power. Power flow analysis is used to determine the steady state operating condition of a power system. In short it is to find the approximate values of various bus voltages, their phase angle, active and reactive power flows through different branches, generators and loads under steady state condition .Heuristic methods are generally referred as experience based techniques for solving problem, learning and discovery. Heuristics are simple and efficient rules coded by evolutionary processes. In this paper, GA, genetic algorithm, one of such heuristic algorithms have been used to do the power flow analysis in a simple six, nine, fourteen, twenty six and thirty bus system.

KEYWORDS : Continuous Genetic Algorithm, Chromosome Crossover, Load Flow Analysis, Newton Raphson Method, Mutation, Multi-Objective Minimization.

\section{INTRODUCTION}

In a three phase ac power system active and reactive power flows from the generating station to the load through different networks buses and branches. The flow of active and reactive power is called power flow or load flow. Power flow studies provide asystematic mathematical approach for determination of various bus voltages, there phase angle active and reactive power flows through different branches, generators and loads under steady state condition. Power flow analysis is used to determine the steady state operating condition of a power system.[1,2,3] Power flow analysis is widely used by power distribution professional during the planning and operation of power distribution system.

- Load-flow studies are performed to determine the steadystate operation of an electric power system. It calculates the voltage drop on each feeder, the voltage at each bus, and the power flow in all branch and feeder circuits.

- Determine if system voltages remain within specified limits under various contingency conditions, and whether equipment such as transformers and conductors are overloaded.

- Load-flow studies are often used to identify the need for additional generation, capacitive, or inductive VAR support, or the placement of capacitors and/or reactors to maintain system voltages within specified limits.

- Losses in each branch and total system power losses are also calculated.

- Necessary for planning, economic scheduling, and control of an existing system as well as planning its future expansion

\section{SYSTEM STUDY METHODOLOGY METHOD}

In power engineering, the power flow analysis (also known as load-flow study) is an importance tool involving numerical analysis applied to a power system. Unlike traditional circuit analysis, a power flow study usually uses simplified notation such as a one-line diagram and per-unit system, and focuses on various form of $\mathrm{AC}$ power (ie: reactive, real and apparent) rather than voltage and current. The advantage in studying power flow analysis is in planning the future expansion of power systems as well as in determining the best operation of existing systems. Power flow analysis is being used for solving power flow problem. There are three methods can be used to solve power flow analysis. The methods are Newton-Raphson method, Fast-Decoupled method and Gauss-Seidel method. This sub-chapter will discuss all three methods generally on formula or mathematical step in order to solve power flow problem.
The methodology of the studies conducted was through theoretical knowledge and system simulation of the distribution grids under study. For system simulation studies, only steady state analysis was done. In theory, understanding of underlying principles of voltage level, profile, line loading, system losses and steady state voltage stability was done for a simple two port system. An extension of theoretical principles was done with power integrated to a simple two port system. Similar studies in other parts of the world and Sweden were looked at to get an insight into likely issues that other scholars have looked at $[1][4,5]$.

Load flow studies are used to ensure that electrical power transfer from generators to consumers through the grid system is stable, reliable and economic. The increasing presence of distributed alternative energy sources, often in geographically remote locations, complicates load flow studies and has triggered a resurgence of interest in the topic. In a three phase ac power system active and reactive power flows from the generating station to the load through different networks buses and branches. The flow of active and reactive power is called power flow or load flow. Power flow studies provide as systematic mathematical approach for determination of various bus voltages, there phase angle active and reactive power flows through different branches, generators and loads under steady state condition. In order to obtain a reliable power system operation under normal balanced three phase steady state conditions, it is required to have the followings:

- Generation supplies the load demand and losses.

- Bus voltage magnitudes remain close to rated values.

- Generator operates within specific real and reactive power limits.

- Transmission lines and transformers are not overloaded. Power flow analysis is used to determine the steady state operating condition of a power system. Power flow analysis is widely used by power distribution professional during the planning and operation of power distribution system

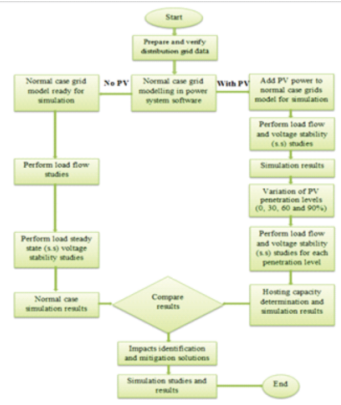

Figure 2.1: System Studies Methodology Flow Chart. 
As shown in Figure 2.1, the comparison of the two cases yielded results that were compared. In these studies, the normal case was considered at maximum load demand of the grids under study. For the cases with power generation, both scenarios of maximum and minimum load demand for the grids were considered. In this process, the hosting capacity of the distribution grids was determined.

\section{POWER/LOAD FLOW STUDIES}

The process of power flow studies is a concept that shows/outlines the flow of power in a grid from one bus to another bus and/or power flow into a bus or leaving the bus. With which shows a two port or bus grid, power was flowing from bus 1 to bus 2 . The power could be seen to enter and leave bus 2. The main aim of a power flow study is to determine power system electrical quantities with some quantities known while others unknown. These quantities are voltage magnitude $(|U|)$, voltage or load angle $(\delta)$, active power (P), reactive power $(\mathrm{Q})$ and apparent power (S). During the process of power flow, line loadings, voltage drops and losses are also determined. To determine unknown electrical quantities to a grid, a load flow analysis needs to be done either manually or with an aid of power system simulation software. One way of determining the voltages (U) and currents (I) at the bus is through the use of bus admittance ( $Y=$ -y) matrix method [3]. This method and circuit analysis yields equations.

$I_{B U S}=Y_{B U S} U_{B U S}$

$\left(\begin{array}{l}I_{1} \\ I_{2} \\ I_{3}\end{array}\right)=\left(\begin{array}{lll}Y_{11} & Y_{12} & Y_{13} \\ Y_{21} & Y_{22} & Y_{23} \\ Y_{31} & Y_{32} & Y_{33}\end{array}\right)\left(\begin{array}{l}U_{1} \\ U_{2} \\ U_{3}\end{array}\right)$

The resultant individual equations from equation for currents and voltages are nonlinear equations. To solve these nonlinear equations, methods of solving non-linear equations and by estimation are used. These are Gauss-Seidel Method, Newton-Raphson and Fast Decoupled methods [6]. These are iteration methods that reduce an error to the first estimated value of the unknown parameter until it is very small. In addition, the power flow into a named bus (i) can be expressed by equation.

$P_{i}-j Q_{i}=U_{i}^{*}\left[U_{i} \sum_{j=0}^{n} y_{i j}-\sum_{j=1}^{n} y_{i j} U_{j}\right]$ where $i \neq j$

Using Gauss-Seidel Method and Newton-Raphson, the equations for power ( $Q$ and $P$ ) and voltage can be written for a three bus grid .These equations are non-linear and use of iteration methods is done to estimate unknown values at the buses. The power system simulation software, Paladin Design base and MATLAB, has the power flow capabilities with the iteration methods mentioned.

Newton-Raphson method is commonly use and introduce in most text book. This method widely used for solving simultaneous nonlinear algebraic equations. A Newton Raphson method is a successive approximation procedure based on an initial estimate of the one-dimensional equation given by series expansion. The Newton-Raphson method using the bus admittance matrix in either first or second order expansion of Taylor series has been evaluate as a best solution for the reliability and the rapid convergence.

\section{POWER/LOAD FLOW}

Studies with Load Profiles Power/load flow with load profiles is load flow study that is an extension of the conventional power/load flow but with an added function of performing the analysis on time based. This analysis is able to determine the bus voltage and power consumption (active and reactive) of a load against a period of time. Power flow studies do not take time into consideration and therefore gives the results of simulation on an instant based scenario. For the thesis study, power flow with load profiles analysis is very important. This is because output from a power is not a constant output but varies depending on the time of the day and the climatic conditions prevailing at that time [7-12]. Additionally, the consumption of active and reactive power for residential and industrial loads equally varies with time. In this analysis, the load profile over a period of time (twenty-four (24) hour period) was defined and power output over the same period of time was defined. A single time load flow calculation was then performed to determine the interaction of the two. The load flow, bus voltages, losses and maximum loads over time (twenty-four (24) hour period) were determined in this way.

Steady State Voltage Stability Voltage stability analysis can be either dynamic or steady state. Dynamic voltage stability takes into account the time simulation of the grid under study. Dynamic voltage stability analysis gives a time sequence of events leading to an instability situation. This gives a much detailed analysis of the collapse that can be used for protection coordination and control. Static or steady state voltage analysis does not take time into account. It gives a state of stability, point of start of instability, nature of problem and some key factors likely to contribute to the phenomena of voltage stability. The static voltage stability is associated with Q-U curves, P-U curves, Q-U sensitivity analysis and Q-U modal analysis [7] [8] [9].

In power engineering, the power flow analysis (also known as load-flow study) is an importance tool involving numerical analysis applied to a power system. Unlike traditional circuit analysis, a power flow study usually uses simplified notation such as a one-line diagram and per-unit system, and focuses on various form of $\mathrm{AC}$ power (ie: reactive, real and apparent) rather than voltage and current. The advantage in studying power flow analysis is in planning the future expansion of power systems as well as in determining the best operation of existing systems. Power flow analysis is being used for solving power flow problem. There are three methods can be used to solve power flow analysis. The methods are Newton-Raphson method, Fast-Decoupled method and Gauss-Seidel method. This sub-chapter will discuss all three methods generally on formula or mathematical step in order to solve power flow problem [13].

V. GAUSS-SEIDEL METHODThe basic power flow equations (4.11) and (4.12) are nonlinear. In an $n$-bus power system, let the number of P-Q buses be $n_{p}$ and the number of P-V (generator) buses be $n_{g}$ such that $n=n_{p}+n_{g}+1$. Both voltage magnitudes and angles of the P-Q buses and voltage angles of the P-V buses are unknown making a total number of $2 n_{p}+$ $n_{q}$ quantities to be determined. Amongst the known quantities are $2 n_{p}$ numbers of real and reactive powers of the $\mathrm{P}-\mathrm{Q}$ buses, $2 n_{g}$ numbers of real powers and voltage magnitudes of the P-V buses and voltage magnitude and angle of the slack bus. Therefore there are sufficient numbers of known quantities to obtain a solution of the load flow problem. However, it is rather difficult to obtain a set of closed form equations. We therefore have to resort to obtain iterative solutions of the load flow problem.

At the beginning of an iterative method, a set of values for the unknown quantities are chosen. These are then updated at each iteration. The process continues till errors between all the known and actual quantities reduce below a pre-specified value. In the Gauss-Seidel load flow we denote the initial voltage of the $i^{\text {th }}$ bus by $V_{i}^{(0)}, i=2, \ldots, n$. This should read as the voltage of the $i^{\text {th }}$ bus at the $0^{\text {th }}$ iteration, or initial guess. Similarly this voltage after the first iteration will be denoted by $V_{i}^{(1)}$. In this Gauss-Seidel load flow the load buses and voltage controlled buses are treated differently. However in both these type of buses we use the complex power equation given in for updating the voltages. Knowing the real and reactive power injected at any bus we can expand as 


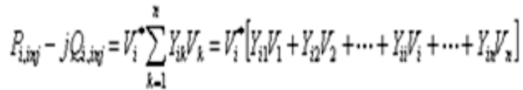

We can rewrite (4.11) as

$V_{i}=\frac{1}{Y_{i i}}\left[\frac{P_{i, i w j}-j Q_{i, i w j}}{V_{i}^{*}}-Y_{i 1} V_{1}-Y_{i 2} V_{2}-\cdots-Y_{i n} V_{n}\right]$

In this fashion the voltages of all the buses are updated. We shall outline this procedure with the help of the system, with the system data given. It is to be noted that the real and reactive powers are given respectively in MW and MVAr. However they are converted into per unit quantities where a base of 100 MVA is chosen.Benefits of Our proposed method:

1. Lower transmission loss.

2. Lower complexity.

3. Applied lower bus data like as $30,60,120$ BUS.

4. Higher Power transmission is possible.

\section{PROPOSED OPTIMIZATTION ALGORITHEM} STEPS IN BASIC GENETIC ALGORITHM

1. [Start] Define the fitness function $\mathrm{f}(\mathrm{x})$ according to the problem definition.

2. [Initialise] Generate random population of $\mathrm{n}$ chromo somes-each chromosome being the potential solution.

3. [Fitness] Evaluate the fitness $\mathrm{f}(\mathrm{x})$ of each chromosome $\mathrm{x}$ in the population.

4. [New population]Repeat the following steps to create the new population of chromosomes:

a. [Selection] Select some parent chromosomes from a population according to their fitness to form mating pool.

b. [Crossover] Mate the selected chromosomes as per given crossover probability to form new off-springs.

c. [Mutation]Mutate new chromosomes as per given mutation probability.

d. [Replace] Replace the old population of chromosomes with the new population.

5. [Convergence check] If the maximum number of generations is reached, then stop, and return the best solution. 6. [Loop] Go to step 3.

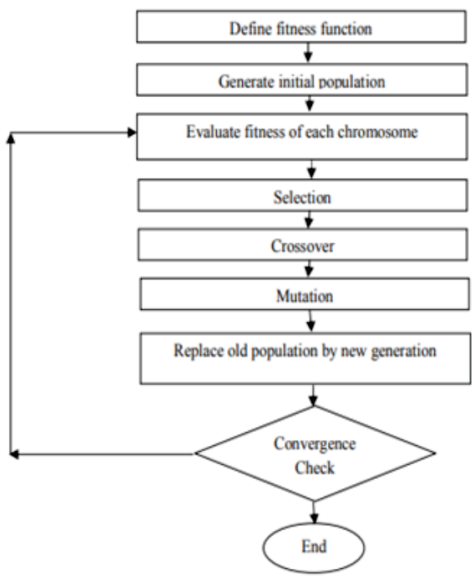

Fig. 6.1: Basic flowchart of Genetic Algorithm

\section{ENCODING OF A CHROMOSOME}

Encoding of chromosomes is the first question to ask when starting to solve a problem with genetic algorithm. A chromosome should in some way contain information about solution that it represents. The most used way of encoding is a binary string. Each chromosome is represented by a binary string (shown in Figure 6.1). Each bit in the string can represent some characteristics of the solution. Another possibility is that the whole string can represent a number. Of course, there are many other ways of encoding. The encoding depends mainly on the solved problem. For example, one can encode directly integer or real numbers, sometimes it is useful to encode some permutations and so on.

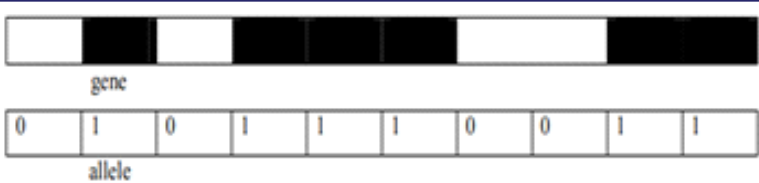

Fig. 6.2: Illustration of Binary Encoded Chromosome SELECTION

Chromosomes are selected from the population to be parents for crossover. The problem is how to select these chromosomes. According to Darwin's theory of evolution the best ones survive to create new offspring. There are many methods in selecting the best chromosomes. Examples are roulette wheel selection, rank selection, steady state selection and some others.

\section{CROSSOVER}

Crossover depends upon the encoding scheme used for the problem. Crossover operates on selected genes from parent chromosomes and creates new offspring. The simplest way of performing crossover is to choose randomly some crossover point and copy everything before this point from the first parent and then copy everything after the crossover point from the other parent. There exist many other ways to perform crossover like n-point crossover, uniform crossover, order crossover etc. Crossover can be quite complicated and depends mainly on the encoding of chromosomes. Specific crossover made for a specific problem can improve performance of the genetic algorithm. One point crossover operation on binary strings is illustrated in Figure6 .3.

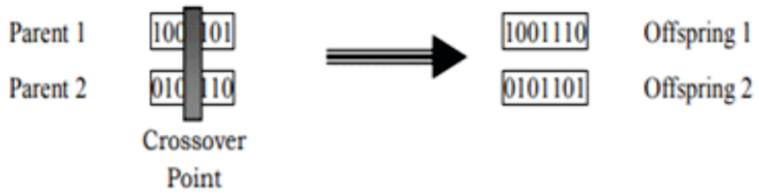

Fig. 6.3: Illustration of Crossover

\section{MUTATION}

After a crossover is performed, mutation takes place. Mutation is intended to prevent falling of all solutions in the population into a local optimum of the solved problem. Mutation operation randomly changes the offspring resulted from crossover. In case of binary encoding we can switch a few randomly chosen bits from 1 to 0 or from 0 to 1 . Mutation can be then illustrated as follows:

\section{Chromosome A}

$101[00101100101011100101$

\section{After Mutation}

101回)0101100101011100101

Fig.6.4: Illustration of Mutation

\section{REPLACEMENT}

When a new generation of off springs is produced, the next question is which of these newly generated off springs would move forward to the next generation and would replace which chromosomes of the current generation. The answer to this question is based on Darwin's principle of "Survival of Fittest". So better fit individuals have more chances to survive and carried forward to next generation leaving behind the less fit ones. The process of forming next generation of individuals by replacing or removing some off springs or parent individuals is done by replacement operator. This process in evolution is known as replacement scheme. Basically, there are two kinds of replacement strategies for maintaining the population generational replacement and steady state replacement. In generational replacement, entire population of genomes is replaced at each generation. In elitism, complete population of genome is replaced except for the best member of each 
generation which is carried over to next generation without modification. In this case, generations are non-overlapping. Steady state replacement involves overlapping population which means only a small fraction of the population is replaced during each iteration. In a steady state replacement, new individuals are inserted in the population as soon as they are created.

\section{PARAMETERS OF GENETIC ALGORITHMS}

A number of parameters control the precise operation of the genetic algorithm. They are:

1. Crossover probability: It is the measure of how often crossover will be performed. If there is no crossover, offspring are exact copies of parents. If there is crossover, offspring are made from parts of both parent's chromosome. If crossover probability is $100 \%$, then all offspring are made by crossover. If it is $0 \%$, whole new generation is made from exact copies of chromosomes from old population. Crossover is made in hope that new chromosomes will contain good parts of old chromosomes and therefore the new chromosomes will be better.

2. Mutation probability: It is the measure of how often parts of chromosome will be mutated. If there is no mutation, offspring are generated immediately after crossover without any change. If mutation is performed, one or more parts of a chromosome are changed. If mutation probability is $100 \%$, whole chromosome is changed, if it is $0 \%$, nothing is changed. Mutation generally prevents the genetic algorithm from falling into local extremes and helps in recovering the lost genetic material. Mutation should not occur very often, because then genetic algorithms would act as to random search.

3. Population size: It is the number of how many chromosomes are present in the population (representing one generation). If there are too few chromosomes, genetic algorithm has few options available for crossover and only a small part of search space is explored. On the counterpart, if there are too many chromosomes in one population then the speed of genetic algorithm slows down.

4. Selection Pressure: Each of the genetic operations crossover, mutation or replacement involves both parent and child chromosomes. The selection of parent chromosomes is biased towards highly fit chromosomes. More fit chromosome is more likely to be a parent than an unfit one in genetic operations. The selection pressure is defined as the ratio between the probabilities that the fit member of the population is selected as a parent to the probability that an average member is selected as a parent. Too high selection pressure would result in the population converging too early, sometimes leading to premature convergence.

5. Number of Operations: The genetic algorithm starts off with a random population of chromosomes. Genetic operations (crossover, mutation, replacement) are then applied iteratively to the population. The parameter number of operations is the number of operators that are applied over the course of a genetic algorithm run.

\section{RESULTS BY GA}

1. We consider 10 initial solutions for each V2, delta2 and delta3 according to their previous experience. For V2 , limits lies in between 0.8to 1.3 and for delta, between 1.00 to -20.00 .

2. Evaluate all the solutions with the fitness function.

3. Select best 5 solutions according to their fitness values along with their repetition to form 10 solutions. 4 . Except first two solutions paired the solutions as parents. Then crossover is done between the parents to get the off springs.

5. Then mutation is performed for some random solution and random parameters to slightly modify the solutions.
6. These contributed to the new generation of ten solutions which includes the offspring and two best solutions.

7. The best two solutions are included to make sure that the best data is available for the next iterations. 8. Now evaluated these 10 solutions and repeated the above procedure till the solution is converged.

9. The converged solution at 5 th iteration was V2 $=1.10200$ delta2 $=-8.00000$ delta $3=-13.0000$ And the error obtained is 1.1038

\begin{tabular}{|l|c|c|c|c|c|}
\hline \multirow{2}{*}{ METHOD } & \multicolumn{5}{|c|}{ BUS SYSTEM LOSS (MW) } \\
\cline { 2 - 6 } & $\mathbf{6}$ & $\mathbf{9}$ & $\mathbf{1 4}$ & $\mathbf{2 6}$ & $\mathbf{3 0}$ \\
\hline Gaussian Seidel & 0.332 & 8.247 & 8.247 & 15.458 & 17.207 \\
\hline Newton Raphson & 1.159 & 8.254 & 12.515 & 15.463 & 17.599 \\
\hline $\begin{array}{l}\text { Optimization Genetic } \\
\text { Algorithm }\end{array}$ & 0.219 & 8.226 & 12.515 & 15.461 & 17.598 \\
\hline
\end{tabular}

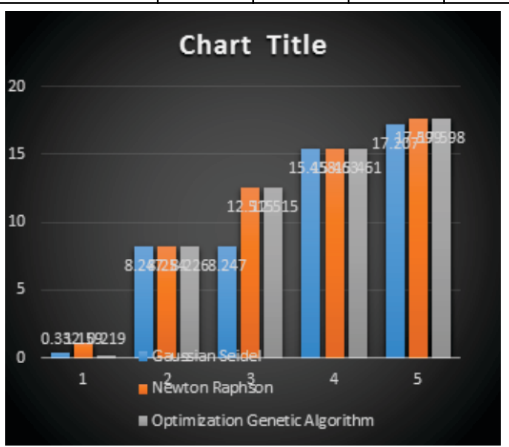

\section{CONCLUSIONS:}

On using the proposed Load Flow Analysis algorithm, considerable amount of saving can be achieved in number of steps execution, required to obtain steady state load flow solutions. Further, on account of algorithm's complexity order; although, the overall complexity of the proposed Load Flow Analysis algorithm is approximately same as one proposed. As for static network topology, the uses of proposed scheme renders saving in time; it indicates that for the network optimization aspects, when network topology undergoes dynamic reconfiguration and thus, this process leads to number of distinct static network topologies. Obviously, for each of these static network topologies, saving in time required for load flow analysis for all these network topologies will be a phenomenal figure. There is significant saving in no. of steps execution as the value of $m$ is very less as compared to the value of $n$.

Future scope: Using graph theory concept and exploiting multi-cores architecture, the proposed method for load flow can be further investigated for obtaining more optimized solutions.

\section{REFERENCES}

[1] A.S. Safigianni, G.N. Koutroumpezis, V.C. Poulios: Mixed Distributed Generation Technologies in a Medium Voltage Network, Electric Power Systems Research, Vol. 96, March 2013, pp. 75-80.

[2] S.M. Moghaddas-Tafreshi, E. Mashhour: Distributed Generation Modeling for Power Flow Studies and a Three-phase Unbalanced Power Flow Solution for Radial Distribution Systems Considering Distribution Generation, Electric Power Systems Research, Vol. 79,No. 4, April 2009, pp. 680-686.

[3] S. Li, K. Tomsovic, T. Hiyama: Load Following Functions using Distributed Energy Resources, IEEE Power Engineering Society Summer Meeting, Seattle, WA, USA, 16-20 July 2000, Vol. 3, pp. 1756-1761

[4] Y. Zhu, K. Tomsovic: Optimal Distribution Power Flow for Systems with Distributed Energy Resources, International Journal of Electrical Power and Energy Systems, Vol. 29, No. 3, March 2007, pp. 260-267.

[5] F.A. Mohamed, H.N. Koivo: System Modeling and Online Optimal Management of Microgrid using Mesh Adaptive Direct Search, International Journal of Electrical Power and Energy Systems, Vol. 32, No. 5, June 2010, pp. $398-407$.

[6] J. Radosavljević, M. Jevtić, D. Klimenta: Optimal Seasonal Voltage Control in Rural Distribution Networks with Distributed Generators, Journal of Electical Engineering, Vol, 61, No. 6, Dec. 2010, pp. 321 -331.

[7] B. Venkatesh: Optimal Power Flow in Radial Distribution Systems, 9th International Power and Energy Conference, Singapore, 27-29 Oct. 2010, pp. $18-21$.

[8] E. Atmaca: An Ordinal Optimization based Method for Power Distribution System Control, Electric Power Systems Research, Vol. 78, No. 4, April 2008, 
pp. $694-702$

[9] A. Gabash P. Li: Active-reactive Optimal Power Flow in Distribution Networks with Embedded Generation and Battery Storage, IEEE Transaction on Power Systems, Vol. 27

[10] A. Borghetti: Using Mixed Integer Programming for the Volt/Var Optimization in Distribution Feeders, Electric Power Systems Research, Vol. 98, May 2013, pp. $39-50$.

[11] B.K. Jo, J.H. Han, Q. Guo, G. Jang: Probabilistic Optimal Power Flow Analysis with Undetermined Loads, Journal of International Council on Electrical Engineering, Vol. 2, No.3, 2012, pp. 321-325.

[12] A. Mohapatra, P.R. Bijwe, B.K. Panigrahi: Optimal Power Flow with Multiple Data Uncertainties, Electric Power Systems Research, Vol. 95, Feb. 2013, pp. $160-167$.

[13] T. Niknam, F. Golestaneh, A. Malekpour: Probabilistic Energy Operation Management of a Microgrid Containing Wind/Photovoltaioc/Fuel Cell Generation and Energy Storage 